\title{
Low-emittance tuning at the Cornell Electron Storage Ring Test Accelerator
}

\author{
J. Shanks, 'D. L. Rubin, and D. Sagan \\ Cornell Laboratory for Accelerator-based Sciences and Education, \\ Cornell University, Ithaca, New York 14853, USA \\ (Received 5 September 2013; published 11 April 2014)
}

\begin{abstract}
In 2008 the Cornell Electron/Positron Storage Ring (CESR) was reconfigured from an electron/positron collider to serve as a test bed for the International Linear Collider damping rings. One of the primary goals of the CESR Test Accelerator (CesrTA) project is to develop a fast low-emittance tuning method which scales well to large rings such as the ILC damping rings, and routinely achieves a vertical emittance of order $10 \mathrm{pm}$ at $2.085 \mathrm{GeV}$. This paper discusses the tuning methods developed at CesrTA to achieve lowemittance conditions. One iteration of beam-based measurement and correction requires about $10 \mathrm{~min}$. A minimum vertical emittance of $10.3(+3.2 /-3.4)^{\text {sys }}( \pm 0.2)^{\text {stat }}$ pm has been achieved at $2.085 \mathrm{GeV}$. In various configurations and beam energies the correction technique routinely achieves vertical emittance around $10 \mathrm{pm}$ after correction, with rms coupling $<0.5 \%$. The measured vertical dispersion is dominated by beam position monitor systematics. The propagation of uncertainties in the emittance measurement is described in detail. Simulations modeling the effects of magnet misalignments, beam position monitor errors, and the emittance correction algorithm suggest the residual vertical emittance measured at the conclusion of the tuning procedure is dominated by sources other than optics errors and misalignments.
\end{abstract}

DOI: 10.1103/PhysRevSTAB.17.044003

PACS numbers: 29.20.db, 29.27.Fh

\section{INTRODUCTION}

In 2008 the Cornell Electron Storage Ring (CESR) was reconfigured from an electron/positron collider to the CESR Test Accelerator (CesrTA) [1-3], a test bed for the International Linear Collider (ILC) damping rings [4]. Parameters for the CESR storage ring are shown in Table I. One of the primary objectives of the CesrTA program is to develop low-emittance tuning methods for the ILC damping rings.

By far the most common tool for linear optics correction is orbit response matrix (ORM) analysis, specifically Linear Optics from Closed Orbits (LOCO) [5,6]. In particular, LOCO has been used as the cornerstone for corrections at both the Swiss Light Source (SLS) and the Australian Synchrotron, where vertical emittances of order $1 \mathrm{pm}$ have been reported [7,8].

However, the time required for measuring the response matrix scales linearly with the number of correctors. The Australian Synchrotron has demonstrated an acquisition rate of order $10 \mathrm{sec}$ per corrector. Assuming the ILC damping rings will be capable of the same acquisition rate, simply measuring the response matrix for all 800 steerings would take several hours, and thus response matrix analysis is deemed prohibitively slow for the ILC damping rings.

\footnotetext{
j3583@cornell.edu
}

Published by the American Physical Society under the terms of the Creative Commons Attribution 3.0 License. Further distribution of this work must maintain attribution to the author $(s)$ and the published article's title, journal citation, and DOI.
The tuning algorithm developed for CesrTA was required to be fast, and scale well to large rings such as the ILC damping rings. The correction procedure takes less than 5 min to acquire a full data set, where the duration time is limited by the slew rate of the superconducting rf cavities for dispersion measurements. One correction iteration (measure, compute corrections, load corrections, and remeasure) takes around $10 \mathrm{~min}$. Data acquisition is fully parallelized, with preprocessing done on beam position monitor (BPM) modules, one for every BPM button. Measurement time for the CesrTA algorithm scales independently of number of BPMs, and does not depend on the number of correctors.

TABLE I. Parameters of the CESR electron/positron storage ring.

\begin{tabular}{lcc}
\hline \hline Parameter & Value & Units \\
\hline Circumference & 768.4 & $\mathrm{~m}$ \\
Energy & $2.085(1.5-5.3)$ & $\mathrm{GeV}$ \\
Lattice type & FODO & \\
Tunes $\left(Q_{x}, Q_{y}\right)$ & $(14.59,9.63)$ & \\
Symmetry & $\approx$ mirror & \\
H/V steerings & $55 / 58$ & \\
Quadrupoles & 105 & \\
Skew quadrupoles & 27 & $\mathrm{~T}$ \\
Damping wigglers & 12 & $\mathrm{~nm}$ \\
Wiggler $B_{\text {max }}$ & 1.9 & $\mathrm{pm}$ \\
Position monitors & 100 & \\
$\epsilon_{x}{ }^{\text {geometric }}$ & 2.7 & \\
$\epsilon_{y}{ }^{\text {geometric }}$ (target) & 10 & \\
\hline \hline
\end{tabular}


The algorithm may also prove useful to other storage rings. Betatron phase measurements are significantly faster than traditional response matrix analyses, allowing for less time to be spent on optics correction. The measurements may be performed using a witness bunch, exciting and measuring only a single bunch in a fully loaded machine. Additionally, measurements such as betatron phase and coupling, which utilize resonant excitation, do not require changing the machine conditions and therefore minimize hysteresis in corrector magnets.

This paper describes the optics correction procedure developed at CesrTA that meets these requirements. Experimental results, with detailed propagation of uncertainties, are presented. Also discussed are simulations of the correction procedure, which have been essential to understanding measurement systematics and recognizing that the residual vertical emittance is dominated by sources other than optics errors and misalignments.

\section{MOTIVATION FOR BEAM-BASED EMITTANCE TUNING}

For the ILC, the quantity of interest is not the projected vertical beam size but rather the emittance $\epsilon_{b}$ of the verticallike normal mode, called the " $b$ mode." In principle, the beam could be intentionally coupled in the damping rings in order to reduce collective effects, and decoupled in the extraction line, so long as the $b$-mode emittance is preserved. The decomposition into normal modes has been discussed elsewhere [9-11], and therefore will not be covered here.

The primary static contributions to $\epsilon_{b}$ in a planar ring are quadrupole tilts, quadrupole vertical offsets, and dipole rolls. Tilted quadrupoles couple horizontal and vertical motion which couples photon emission in the horizontal plane to the $b$ mode. Vertical quadrupole offsets and dipole rolls introduce vertical kicks, generating vertical dispersion and thus vertical emittance. Additional sources of vertical emittance include time-dependent variations associated with line voltage, ground motion, and feedback systems, which contribute kicks to the beam in various ways, and currentdependent effects such as intrabeam scattering (IBS).

Without beam-based corrections of dispersion and coupling, the vertical emittance would be limited by the quality of survey and alignment. Survey and alignment is accomplished primarily using a Leica AT402 absolute laser tracker for establishing the reference network and a Leica DNA03 digital level for elevation runs. Establishing the reference network is done via free stationing, with over 100 stations in the network. The reference network consists of a triplet of reference targets - attached to the inner tunnel wall, outer tunnel wall, and embedded in the floorapproximately every $8-10 \mathrm{~m}$ in the tunnel arcs, and less regular, but on average equally dense wall, floor, and ceiling targets in the long-straight sections, flares, and main south areas. Typical combined (bundled) $1 \sigma$ uncertainties

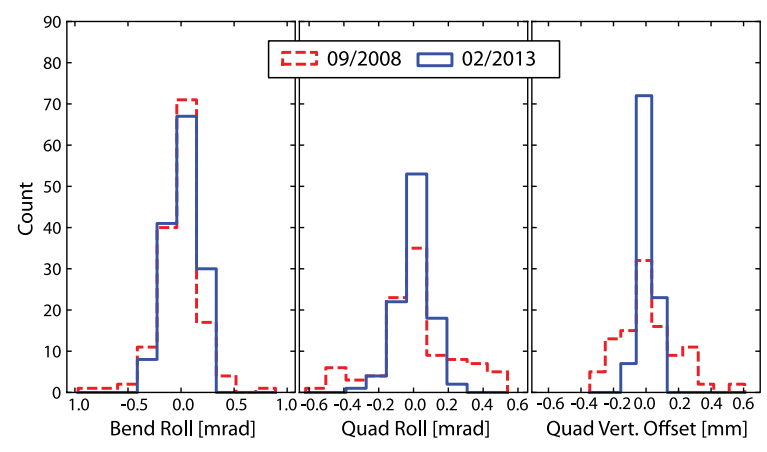

FIG. 1. Survey and alignment results for CesrTA as of December 2012 CesrTA run, compared to alignment in September 2008 at the start of the CesrTA program. Left to right: dipole roll, quadrupole tilt, and quadrupole vertical offset.

for the reference targets are on order $35 \mu \mathrm{m}$ for height $(z)$. Magnets are surveyed into place to better than $100 \mu \mathrm{m}$ of measured versus ideal positions using fiducials mechanically aligned to their irons. The reference network and magnets are resurveyed regularly. Comparisons of reference target variations from survey to survey establish the uncertainties used in the analysis.

The measured distributions of surveyed quadrupole and dipole offsets and tilts for CESR are shown in Fig. 1. The root mean square (rms) of the position and tilt measurements are summarized in Table VIII in the Appendix, and include an estimated $100 \mu \mathrm{m}$ uncertainty in the displacement of the magnetic center from physical center of the magnet.

Simulations using random distributions of magnet, beam position monitor, and multipole errors consistent with measurements (summarized in the Appendix) have been
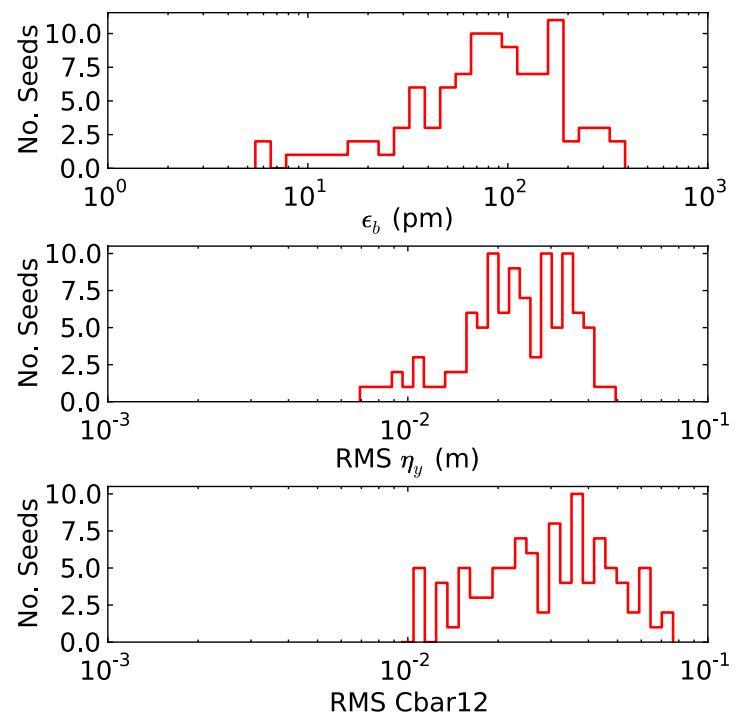

FIG. 2. Resulting distributions of vertical emittance, vertical dispersion, and coupling when applying random distributions of errors at the amplitudes specified in Table VIII, along with systematic and random multipoles specified in Table IX. 
used to study the effect of these errors on the vertical emittance. Repeating for 100 random sets of magnet errors, the resulting distributions of emittance, dispersion, and coupling yield statistical information about the probability of achieving the target emittance, and are shown in Fig. 2. The coupling is characterized using the $\bar{C}$ coupling matrix, an extension of the Edwards and Teng formalism [12] and defined in [13]. In particular, only the out-of-phase coupling matrix element $\bar{C}_{12}$ is considered, for reasons discussed in Sec. III.

Without any beam-based corrections, simulations show that out of 100 random seeds, only three yielded the target vertical emittance of $10 \mathrm{pm}$; the mean vertical emittance of the 100 seeds is $104 \mathrm{pm}$. It is evident that the survey and alignment techniques used are insufficient by themselves to reach the CesrTA emittance target. Some form of beambased correction is clearly required in order to achieve and maintain low-emittance operating conditions.

\section{MEASUREMENT TECHNIQUES}

Beam position monitors (BPMs) are used to collect data for most beam-based optics characterization techniques used in emittance tuning at CesrTA. CESR is instrumented with 100 button-style peak-detection BPMs. A cross section of a typical CESR BPM is shown in Fig. 3. New electronics, developed in-house, were installed in 2009 [14]. The new BPM system is capable of bunch-bybunch, turn-by-turn readout for bunch spacings $\geq 4$ ns with a buffer of 300,000 bunch turns. At each BPM, all four button channels are read out by separate controller cards; therefore, channel-to-channel crosstalk is minimized. Bunch-to-bunch cross-talk is below $4 \%$ after $4 \mathrm{~ns}$, and is effectively zero after $50 \mathrm{~ns}$; there is no turn-to-turn crosstalk. Single-turn orbit reproducibility is measured to be $7 \mu \mathrm{m}$ for consecutive turns; as this is determined from beam-based measurements, it includes not only the

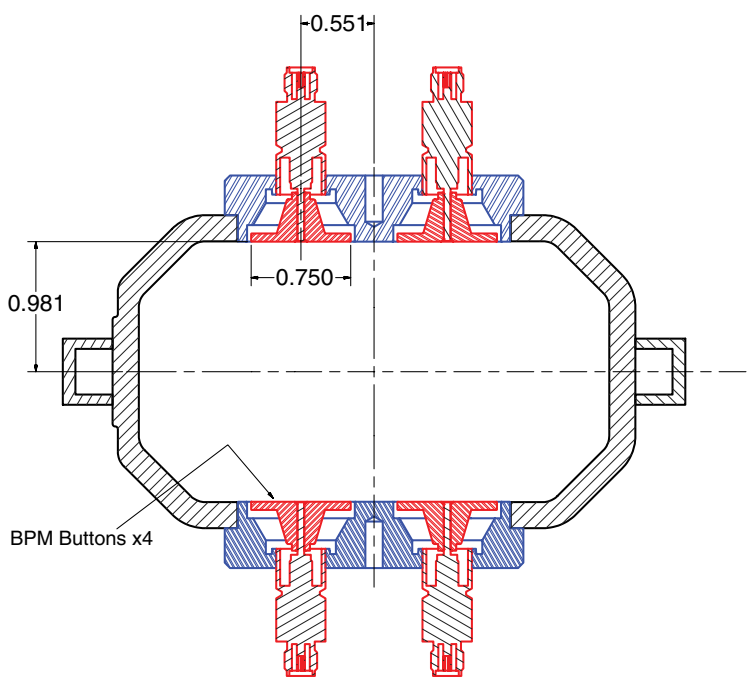

FIG. 3. Cross section of a CESR BPM. Dimensions are in inches. sensitivity of the BPM, but also all contributions such as electronic stability and beam pipe vibration. Depending on the user's request for data, some level of preprocessing is done onboard the BPM electronics before committing data to file, or the raw bunch-by-bunch turn-by-turn button signals are written directly to file.

BPMs are used to measure closed orbit, betatron phase and coupling, and dispersion. Turn-by-turn trajectory data is also used for BPM calibrations. For all beam-based measurements in low-emittance tuning at CesrTA, a single bunch of $0.8 \mathrm{~mA}=1.3 \times 10^{10}$ particles is used. Therefore, bunch-to-bunch effects do not contribute to emittance measurements.

The closed orbit at each BPM is measured by averaging 1024 turns of turn-by-turn bunch trajectory data onboard the BPM modules. A closed orbit measurement takes roughly $5 \mathrm{sec}$, with measurement reproducibility of around $2 \mu \mathrm{m}$.

Dispersion measurements are performed in the usual way, by varying the frequency of the rf cavities by a known amount, which changes the beam energy, and measuring the change in closed orbit. A standard dispersion measurement at CESR varies the $500 \mathrm{MHz}$ cavities by $\pm 2 \mathrm{kHz}$ (corresponding to $\delta_{E} / E \pm 5.9 \times 10^{-4}$ ) and takes several minutes to acquire. The measurement time is determined by the slew rate of the frequency of the superconducting rf cavities. The measurement reproducibility is better than $5 \mathrm{~mm}$.

Quadrupole focusing errors are determined by measuring betatron phase advance at each BPM, using turn-by-turn data acquired while resonantly exciting the beam [15]. Resonant excitation is achieved through a pair of "tune trackers," which are stripline kickers phase locked to the horizontal and vertical betatron tunes [16]. The tune trackers excite the beam to amplitudes of several millimeters. Phase and amplitude data are extracted from 40,960 consecutive turns by a processor onboard the BPM module for each button. The button-by-button phase and amplitude are postprocessed into horizontal and vertical phase, the out-ofphase component of the coupling matrix $\bar{C}_{12}$, and the two in-phase components of the coupling matrix $\bar{C}_{22,11}$. All of the above information is processed from one measurement of the machine. Betatron phase data for all 100 BPMs is collected and analyzed in $10 \mathrm{sec}$. Reproducibility of betatron phase measurements is of order $0.1^{\circ}$.

When characterizing coupling, only the coupling matrix element $\bar{C}_{12}$ is used, and the other two measured components $\left(\bar{C}_{22}\right.$ and $\left.\bar{C}_{11}\right)$ are neglected. It is not possible to measure $\bar{C}_{21} . \bar{C}_{12}$ represents the out-of-phase propagation of coupling (a sinelike vertical motion at the horizontal tune, when the horizontal is excited with a cosinelike signal), and it can be shown that $\bar{C}_{12}$ is insensitive to BPM rotations. Second, two independent measurements of $\bar{C}_{12}$ can be made simultaneously, from excitations of the horizontal and vertical modes, adding redundancy in the measurement. Third, because the (normalized) coupling 
matrix elements mix from one BPM to the next, measuring and correcting $\bar{C}_{12}$ globally is sufficient to correct the entire coupling matrix.

For clarity it is perhaps worth comparing the betatron phase and coupling technique with ORM measurement and analysis. The response matrix is established by measurement of the closed orbit (position) at each BPM in response to excitation of the distributed steering corrector magnets. From the set of measured closed orbits (two for each steering), the linear transfer matrix (betatron phase advance and coupling) from one BPM to the next may be reconstructed.

In the phase and coupling measurement as implemented for CESR, the transfer matrices are similarly reconstructed from measured trajectories. The turn-by-turn capability of the beam position monitors is exploited to significantly reduce the measurement time. Rather than drive DC correctors to generate a distribution of trajectories, the beam is resonantly excited at the betatron tunes by a single source at a fixed location. As the tunes are noninteger, the 40,960 consecutive trajectories smoothly sample phase space, and are acquired in a fraction of a second. The trajectories could in principle be analyzed using ORM techniques. Alternatively (and equivalently), we extract betatron phase and amplitude and coupling information with the help of preprocessing in each of the BPM modules. The objective for the emittance correction method at CesrTA is a technique suitable for a ring with very large circumference, like the ILC damping ring; therefore, the betatron phase and coupling technique is favored.

\section{BPM CALIBRATIONS}

In order to ensure that measurements reflect actual machine conditions, BPMs must be well calibrated. The primary characteristics to consider are button-by-button timing, button-to-button relative gains, BPM tilts, and BPM-to-quadrupole transverse offsets.

Many modern lightsource BPMs take four signals into one controller that preprocesses the raw signals into horizontal and vertical data. CESR BPMs have four separate controller cards, one for each button, which read out independently. This allows for greater flexibility in measurements and postprocessing; however, some characteristics such as timing and gains must be calibrated on each of the four button channels rather than once per BPM.

Each of the required calibrations are now discussed in the order of implementation.

\section{A. BPM timing}

Each controller card has independent timing; therefore, every button on every BPM must be timed in separately. A mistimed channel results in sampling the bunch passage off-peak, which reduces the observed signal amplitude for that button.

The time-in procedure consists of sampling the temporal profile of a bunch passage at a resolution of $10 \mathrm{ps}$ and fitting to determine the peak. The process converges in less than one minute for all four buttons on all 100 BPMs, with less than 10 ps drift over a period of $4 \mathrm{hr}$.

\section{B. Button-to-button relative gains}

Differential response of the four BPM buttons due to variations in relative electronic gain will introduce a systematic measurement error. Measurements that depend mostly on position, such as orbit, dispersion, and the in-phase components of the coupling matrix $\bar{C}_{22,11}$, are sensitive to relative button gains. Measurements using relative phase, such as betatron phase advance and the out-of-phase coupling matrix element $\bar{C}_{12}$, are largely insensitive to gain errors.

The method of gain mapping used at CesrTA was developed by Rubin et al. at Cornell [17] and is based on a second-order expansion of the button signal response. The method utilizes turn-by-turn data; therefore, data acquisition is fast, on the order of several seconds to collect data for characterizing all 100 BPMs.

The analysis relies on the fact that a linear relation exists between two combinations of the four button signals. For $n$ turns of turn-by-turn trajectory data there are $4 n$ button measurements at each BPM. There are only four unknowns, namely, the button gains, and the system is overconstrained for $n>1$ orbits; typically 1024 turns are used. Data acquisition takes about $10 \mathrm{sec}$, and the fitting process takes less than a minute to determine all four button gains on all 100 BPMs.

All gain calibration techniques for peak-detection-style BPMs are sensitive to timing errors. This method is insensitive to detector rotation or offset, as the method uses raw button signals across a large cross section of the BPM, and does not rely on distinguishing between horizontal and vertical modes.

Typical BPM gain variations before correction are of order $5 \%$, and are calibrated with a reproducibility of a few tenths of a percent.

\section{BPM electronic centering}

A relative offset between the electronic center of a BPM and the magnetic center of the nearest quadrupole will appear in measurements as an offset in the quad. If the relative offset is not calibrated, steering the beam to the electronic center of the BPM will result in kicks from the quadrupole, generating dispersion. To minimize vertical dispersion (and thus the emittance) generated during orbit correction, the relative offset between the electronic center of a BPM and the magnetic center of the nearest quadrupole must be measured.

The method used at CESR for determining the BPM-toquadrupole transverse offset is based on a common technique where the beam is steered such that a change in the quadrupole strength results in no change in orbit $[18,19]$. The method employed at CESR has the additional benefit that the change in quadrupole strength $K_{1}$ is 
determined using betatron phase measurements [20]. By measuring the difference in phase before and after the quadrupole change, the change in quadrupole strength can be more accurately determined than using the change in quadrupole current, which is susceptible to hysteresis. Therefore, fewer iterations are required on each BPM/ quadrupole pair to achieve convergence.

Typical horizontal and vertical BPM-to-quadrupole offset measurements are around $1 \mathrm{~mm}$ rms, with a short-term reproducibility of order $50 \mu \mathrm{m}$ and long-term drift of about $110 \mu \mathrm{m}$ over the course of a 3 -week machine studies period.

BPM-to-quadrupole relative centering will only affect orbit measurements and turn-by-turn trajectory data. Dispersion measurements are a difference of two closed orbits; therefore, absolute offsets do not affect the measurement. Betatron phase and coupling measurements are computed button by button; therefore, transverse offsets will not affect the measurement.

\section{BPM tilt calibration}

If a BPM is rotated, a horizontal orbit perturbation will indicate a vertical offset. This becomes particularly significant when measuring the dispersion, as the average horizontal dispersion in CESR is large, on the order of a meter. The lowest vertical dispersion measured without BPM tilt corrections is around $12 \mathrm{~mm} \mathrm{rms}$. The measurements cannot be fit with a model dispersion function generated by corrector magnets or magnet misalignments. Furthermore, simulations have shown that a rms of $12 \mathrm{~mm}$ of actual vertical dispersion corresponds to $20-30$ pm vertical emittance, significantly larger than the emittance determined from vertical beam size measurements. This implies the measurement is dominated by systematic errors, such as uncalibrated BPM tilts. If the BPM tilts are uncorrelated with the horizontal dispersion, this constrains the distribution of BPM tilts to have a maximum rms of $12 \mathrm{mrad}$.

BPM tilts can in principle be determined by linearly fitting turn-by-turn trajectory data for a well-decoupled beam that is resonantly excited in the horizontal mode. Residual in-phase coupling will also rotate the beam in $x-y$ space, which introduces a lower bound on the ability to resolve BPM tilts using this method to around $5 \mathrm{mrad}$. To date, applying the BPM tilts to dispersion data does not improve the ability to fit the vertical dispersion function. Several alternative methods for measuring BPM tilts have been explored, all yielding different calibrations, and none improving the ability to fit the vertical dispersion. As such, the tilt calibrations have not been utilized during any of the corrections described in this paper, and remain the most significant known systematic in optics corrections.

\section{BEAM SIZE INSTRUMENTATION-XBSM}

The primary method of determining the effectiveness of vertical emittance tuning is direct observation of the vertical beam size, from which the emittance can be inferred. CESR is instrumented with two $\mathrm{x}$-ray beam size monitors (xBSM), one for each species $[21,22]$.

The xBSMs are one-dimensional 32-diode arrays with $50 \mu \mathrm{m}$ pixel pitch. The instruments are capable of bunchby-bunch, turn-by-turn measurements with a buffer of 250,000 bunch turns. Dynamic range for the instruments span beam currents $0.25-10 \mathrm{~mA}=0.4-16 \times 10^{10} /$ bunch at the standard CesrTA operating energy of $2.085 \mathrm{GeV}$.

When characterizing low-emittance conditions, the beam is typically imaged using a horizontal slit formed by two tungsten blades, which act as a one-dimensional pinhole. Beam size is determined by fitting to the beam profile over 1024 turns on a turn-by-turn basis, then averaging. In this way any effect of turn-by-turn beam centroid or x-ray optics motion is removed from the measured beam size. The fitting procedure takes into account the point-response function of the imaging device (in this case, the onedimensional pinhole), including the effects of the finite opening angle, depth of field, energy spectrum, diffraction off the tungsten blades, surface roughness of the tungsten blades, and detector response. A detailed analysis of the fitting procedure is available in [23]. In practice, the resolution limit when using the pinhole optics is around $10-15 \mu \mathrm{m}$. The vertical beta function $\beta_{b}$ at the xBSM source point is approximately $40 \mathrm{~m}$, and the xBSM optics provide a magnification of approximately 2.2 . Therefore, the xBSMs are able to resolve the vertical emittance down to $2.5-5.5 \mathrm{pm}$.

\section{LOW-EMITTANCE TUNING}

The low-emittance tuning procedure developed at CesrTA takes advantage of the fact that all magnets are independently powered, and all BPMs are capable of betatron phase and coupling measurements through turn-by-turn acquisition. The procedure is as follows:

1. Measure the closed orbit and correct to a reference orbit (which aligns the beam with the xBSM beamline) using all 55 horizontal and 58 vertical steering correctors.

2. Measure the betatron phase, transverse coupling $\left(\bar{C}_{12}\right)$, and horizontal dispersion. Fit the model lattice to the measurement using all 100 quadrupoles and 27 skew quadrupole correctors, and load the computed corrections.

3. Remeasure the closed orbit, transverse coupling, and vertical dispersion. Fit the model lattice to all machine data simultaneously using all vertical steerings and skew quadrupoles, and load the fitted corrector changes.

The turnaround time for one full set of corrections is roughly $10 \mathrm{~min}$. It is standard procedure when first recovering conditions to save magnet settings after achieving low emittance, run the machine through a well-defined hysteresis loop, reload the previously saved conditions, and repeat the 
TABLE II. Typical levels of correction for optics measurement after the full emittance tuning procedure. Measurements were taken at $0.8 \mathrm{~mA}\left(1.3 \times 10^{10} / \mathrm{bunch}\right)$. Root mean square deviations from the design are reported for both the machine measurement and a model of the machine that is fit to the measurements. Beta beat is computed from fitting phase data.

\begin{tabular}{lccc}
\hline \hline Measurement & rms (data) & rms (model) & Units \\
\hline$\delta y$ & 253 & 110 & $\mu \mathrm{m}$ \\
$\delta \phi_{a, b}$ & 0.3 & 0.3 & $\mathrm{deg}$ \\
$\delta \beta / \beta$ & Not measured & $0.73 \%$ & $\%$ \\
$\eta_{y}$ & 13 & 5 & $\mathrm{~mm}$ \\
$C_{12}$ & 0.004 & 0.003 & \\
\hline \hline
\end{tabular}

emittance tuning procedure to apply minor corrections and ensure the desired conditions are reproducible.

Lattice corrections are determined by a $\chi^{2}$ minimization where a machine model is fit to measurements of the lattice functions, with a merit function defined as [15]

$$
\begin{aligned}
\chi^{2}= & \sum_{i} w_{i}^{\text {data }}\left[d^{\text {measured }}(i)-d^{\text {model }}(i)\right]^{2} \\
& +\sum_{j} w_{j}^{\operatorname{var}}\left[v^{\text {measured }}(i)-v^{\text {model }}(i)\right]^{2},
\end{aligned}
$$

where $d(i)$ is the $i$ th datum (for example, the vertical orbit at a BPM), $v(j)$ is the $j$ th variable (such as a corrector strength), and $w_{i, j}$ are user-defined weights. The merit function is minimized by adjusting corrector magnets in the model such that the model reproduces the measurements. The machine model corrector strengths are then loaded into the machine with opposite sign to compensate for optics errors.

Beam-based characterization of the machine after a typical low-emittance correction is shown in Table II. The discrepancy between the model that best fits those measurements and the design demonstrates the effectiveness of the correction.

The $b$-mode emittance is determined from measurements of the beam size and local optics at the beam size monitor source point:

$$
\epsilon_{b}=\frac{\sigma_{y, b}^{2}}{\gamma_{c}^{2} \beta_{b}}
$$

where $\gamma_{c}$ is a parameter related to the coupling matrix and is approximately unity when coupling is small. $\sigma_{y, b}$ is the contribution from the $b$ mode to the vertical beam size, which is computed from the total vertical beam size $\sigma_{y}$ :

$$
\begin{gathered}
\sigma_{y}=\sqrt{\sigma_{y, a}^{2}+\sigma_{y, b}^{2}+\sigma_{y, \eta_{y}}^{2}}, \\
\sigma_{y, a}=\sqrt{\epsilon_{a} \beta_{b}}\left[\bar{C}_{22}^{2}+\bar{C}_{12}^{2}\right]^{1 / 2}, \\
\sigma_{y, \eta_{y}}=\eta_{y} \frac{\sigma_{E}}{E},
\end{gathered}
$$

where $\sigma_{y, a}, \sigma_{y, b}$, and $\sigma_{y, \eta_{y}}$ are the contributions to the vertical beam size from the horizontal emittance, vertical emittance, and vertical dispersion. $\epsilon_{a}$ is the horizontal-like normal mode. $\bar{C}_{22}$ and $\bar{C}_{12}$ are in-phase and out-of-phase components of the coupling matrix, respectively, and are directly measured at BPMs adjacent to the source point. Equations (3)-(5) are used to determine the component of the vertical beam size due to $b$-mode emittance, $\sigma_{y, b}$, which is then used in Eq. (2).

$\eta_{y}, \beta_{b}, \bar{C}_{22}$, and $\bar{C}_{12}$ are determined by fitting a model of the accelerator to measurements. The beam size at the source point $\sigma_{y}$ is calculated from the measured image at the xBSM $\sigma_{i m}$, accounting for the magnification, energy spectrum, and point-source response of the optic and detector system.

Statistical and systematic errors associated with measurements of vertical emittance with the xBSM are outlined in [24] and include contributions from turn-by-turn beam size fitting uncertainty; turn-by-turn beam size fluctuation; uncertainty in pinhole size; uncertainty in $\beta$ functions; uncertainty in longitudinal location of the $\mathrm{x}$-ray source point; and uncertainty in dispersion at the source point. The uncertainties propagate as follows:

$$
\begin{aligned}
\delta \epsilon_{b}^{\text {sys }} & =\left|\frac{d \epsilon_{b}}{d \sigma_{i m}}\right| \delta \sigma_{i m}^{\text {sys }}+\left|\frac{d \epsilon_{b}}{d \sigma_{p}}\right| \delta \sigma_{p}+\left|\frac{d \epsilon_{b}}{d s}\right| \delta s, \\
\delta \epsilon_{b}^{\text {stat }}= & \left(\left|\frac{\partial \epsilon_{b}}{\partial \beta_{b}}\right|^{2}\left(\delta \beta_{b}^{\text {stat }}\right)^{2}+\left|\frac{\partial \epsilon_{b}}{\partial \eta_{y}}\right|^{2}\left(\delta \eta_{y}^{\text {stat }}\right)^{2}\right. \\
& +\left|\frac{\partial \epsilon_{b}}{\partial \bar{C}_{22}}\right|^{2}\left(\delta \bar{C}_{22}^{\text {stat }}\right)^{2}+\left|\frac{\partial \epsilon_{b}}{\partial \bar{C}_{12}}\right|^{2}\left(\delta \bar{C}_{12}^{\text {stat }}\right)^{2} \\
& \left.+\left|\frac{\partial \epsilon_{b}}{\partial \sigma_{i m}}\right|^{2}\left(\delta \sigma_{i m}^{\text {stat }}\right)^{2}\right)^{1 / 2},
\end{aligned}
$$

where

$$
\left|\frac{d \epsilon_{b}}{d s}\right|=\left|\frac{\partial \epsilon_{b}}{\partial \beta_{b}} \frac{\partial \beta_{b}}{\partial s}+\frac{\partial \epsilon_{b}}{\partial \bar{C}_{22}} \frac{\partial \bar{C}_{22}}{\partial s}+\frac{\partial \epsilon_{b}}{\partial \bar{C}_{12}} \frac{\partial \bar{C}_{12}}{\partial s}+\frac{\partial \epsilon_{b}}{\partial \eta_{y}} \frac{\partial \eta_{y}}{\partial s}+\frac{\partial \epsilon_{b}}{\partial M} \frac{\partial M}{\partial s}\right|
$$

and sys and stat refer to the systematic and statistical uncertainties, respectively. The individual terms $d \epsilon_{b} / d x_{i}$ are computed by varying the terms $x_{i}$ in the emittance calculation by their uncertainties $\pm \delta x_{i}$. 
TABLE III. Lowest-achieved vertical emittance at CesrTA in a variety of energies. Electron conditions are only reported for the April 2013 CesrTA run at $2.085 \mathrm{GeV}$.

\begin{tabular}{lcccc}
\hline \hline Energy $[\mathrm{GeV}]$ & Species & $\epsilon_{b}(\mathrm{pm})$ & $\delta \epsilon_{b}^{\text {sys }}(\mathrm{pm})$ & $\delta \epsilon_{b}^{\text {stat }}(\mathrm{pm})$ \\
\hline $2.085(03 / 2013)$ & $e^{+}$ & 10.3 & $\left\{\begin{array}{l}+3.2 \\
-3.4\end{array}\right.$ & $\left\{\begin{array}{l}+0.2 \\
-0.2\end{array}\right.$ \\
$2.085(03 / 2013)$ & $e^{-}$ & 13.3 & $\left\{\begin{array}{l}+3.3 \\
-3.4\end{array}\right.$ & $\left\{\begin{array}{l}+0.3 \\
-0.3\end{array}\right.$ \\
$2.305(12 / 2012)$ & $e^{+}$ & 10.0 & $\left\{\begin{array}{l}+2.8 \\
-3.7\end{array}\right.$ & $\left\{\begin{array}{l}+0.2 \\
-0.2 \\
+2.9\end{array}\right.$ \\
$2.553(03 / 2013)$ & $e^{+}$ & 10.2 & $\left\{\begin{array}{l}+0.2 \\
-3.4\end{array}\right.$ & $\left\{\begin{array}{l} \\
\hline \hline\end{array}\right.$
\end{tabular}

Using the above tuning method, and propagating errors according to Eqs. (7) and (8), the vertical emittances achieved at CesrTA are reported in Table III.

These results warrant a few comments. First, all four reported measurements are within $1 \sigma$ of the target $\epsilon_{b}<10 \mathrm{pm}$. It is also interesting to note that the minimum-achieved emittances are independent of energy. All four measurements are within $1 \sigma$ of each other, with only the single electron measurement standing out.

The component of the observed vertical beam size due to local coupling $\left(\sigma_{y, a}\right)$ only introduces $0.5 \mu \mathrm{m}$ in quadrature. The observed beam size is of order $20 \mu \mathrm{m}$, and as such, the contribution of local coupling is insignificant.

It is also worth reminding the reader that the minimum measured $\mathrm{rms} \eta_{y}$ of $12 \mathrm{~mm}$ corresponds to a vertical emittance of 20-30 pm, much larger than what has been measured. It is clear that the dispersion measurement is dominated by systematics, and in particular, BPM tilts must be better understood. However, correcting the vertical orbit and transverse coupling indirectly reduces the vertical dispersion. The procedure therefore results in a vertical dispersion below the present resolution of the measurement.

Several alternative emittance tuning methods have been explored, including ORM analysis [25] and normal-mode analysis [26]. To date, no method has proven to be faster or yield consistently better results than the three-stage correction algorithm based on betatron phase and coupling measurements discussed here.

\section{EMITTANCE TUNING SIMULATIONS}

To better characterize what factors are limiting emittance corrections, software has been developed to evaluate the contributions of misalignments, BPM measurement errors, and choice of correction procedure. The program, RING_MA2, uses the BMAD accelerator code library [27] and does the following:

1. Assigns random misalignments and BPM errors with user-defined amplitudes to the ideal lattice to create a realistic machine model.

2. Simulates beam-based measurements of optics functions including the effects of BPM measurement errors.
3. Computes and applies corrections for each iteration based on the simulated measurements.

4. After each correction iteration, it records the effectiveness of the correction in terms of emittances and optics functions.

The entire procedure is repeated typically 100 times in order to generate statistics for analysis. The simulation is approached from a statistical perspective for three reasons. First, magnet positions continually drift, making it difficult to know the exact set of misalignments in the ring on any given day. Second, the precise distributions of magnetic centering or BPM measurement errors are not known, mandating that their distributions be approached from a statistical perspective. Third, by framing the analysis in terms of statistical probability of achieving the required emittance, the characterization process may be extrapolated to new machines that are not yet built using only the knowledge of survey and alignment tolerances.

When discussing the results of statistical analysis, the 95\% confidence levels (C.L.) are presented. That is, after applying the full optics correction procedure, $95 \%$ of simulated lattices, each with a randomly chosen distribution of misalignments and measurement errors, achieve a vertical emittance below the $95 \%$ C.L. The simulation is believed to be sufficiently complete such that it is very unlikely that the contribution of the static optics to the vertical emittance is greater than this number.

In this section the method for simulating optics measurements is discussed, including how BPM measurement errors and guide field magnet errors are modeled. Results of simulations based on input parameters representing the physical accelerator are given.

\section{A. Model lattice with errors}

BMAD allows for introducing strength errors (including systematic and random multipole errors) and alignment errors (such as offset, roll, and pitch) to any lattice element. Magnet strength errors scale with the absolute strength of the magnet. Alignment errors are treated as additive errors, and are applied directly without scaling.

RING_MA2 also models BPM measurement errors, which are discussed in detail in Sec. VII C.

\section{B. Simulated measurements}

All simulated measurements are modeled as realistically as possible. For closed orbit measurements this involves recording 1024 turns of trajectory data, including the effects of BPM measurement errors on every turn, and averaging the results. Dispersion is simulated as a difference of two closed orbits, varying the radio frequency in between.

For phase and coupling measurements, a particle is resonantly excited using a simulated phase-locked tune tracker and allowed to equilibrate by tracking for several damping times ( $10^{5}$ turns). After the particle trajectory has 
TABLE IV. rms difference between simulated measurements and BMAD-calculated values, neglecting any BPM measurement errors.

\begin{tabular}{lcc}
\hline \hline Measurement & rms (simulated-BMAD) & Units \\
\hline Closed orbit $x, y$ & $0.70,<1 \times 10^{-3}$ & $\mu \mathrm{m}$ \\
$\eta_{x, y}$ & $0.75,<1 \times 10^{-6}$ & $\mathrm{~mm}$ \\
$\phi_{x, y}$ & $0.1,0.05$ & $\mathrm{deg}$ \\
$\bar{C}_{12}$ & $4.3 \times 10^{-4}$ & \\
\hline \hline
\end{tabular}

equilibrated, 40,960 turns of raw BPM button data are recorded at every BPM. The data is then processed with the same code used for processing CESR phase and coupling data.

A comparison of lattice parameters derived from simulated measurements in an ideal lattice and those computed directly are summarized in Table IV for each measurement type, and presumably represent a fundamental lower limit to the resolution of each measurement technique given no errors in the BPM measurements. Simulated measurements have differing levels of agreement for horizontal and vertical, which can be attributed to energy loss from stochastic radiation emission, leading to a "sawtooth" horizontal dispersive orbit between the two pairs of rf cavities on opposite sides of the ring. This effect is not seen in the vertical as there is no vertical dispersion in the design lattice.

\section{BPM errors}

To generate simulated measurements as realistically as possible, BPM measurement errors must be taken into account. The two classes of BPM errors modeled in RING_MA2 are BPM misalignments (offsets, tilts, and shear) and button-by-button effects (button gain, timing, and electronic noise). Each class of errors will affect the measurement differently. All simulated measurements presented include the effects of all listed BPM measurement errors.

\section{BPM misalignments}

Errors in BPM misalignments (offsets and tilts) are applied in the following way:

$$
\left(\begin{array}{l}
x \\
y
\end{array}\right)^{m}=R(\theta)\left(\begin{array}{c}
x^{l a b}-\delta x \\
y^{l a b}-\delta y
\end{array}\right)
$$

where $(x, y)^{m}$ are the coordinates with BPM misalignments applied, $R(\theta)$ is the rotation matrix for angle $\theta$, and $\delta x, \delta y$ are the horizontal and vertical offset between the BPM and nearest quadrupole.

\section{Button effects: Gain, timing, and reproducibility}

Timing errors, gain variations, and turn-by-turn resolution affect individual button signals. Modeling their effects requires an accurate method for converting from $(x, y)$ coordinates to button signals $b_{1,2,3,4}$, applying errors, and converting back to $(x, y)$ coordinates.

All button-by-button errors of these classes are handled through use of a nonlinear interpolation grid which converts $(x, y)$ coordinates to button signals. Button-by-button errors are applied to the individual channels, and the final "measured" $(x, y)$ coordinates are determined by the best fit to the set of new button signals using the same interpolation grid [28]. The nonlinear map used in these studies is for a BPM with a "CESR geometry" (see Fig. 3).

Including effects from button-to-button gain errors, timing errors, and measurement reproducibility, the four observed button signals $b_{i}$ at each BPM are

$$
b_{i}^{\text {meas }}=g_{i} t_{i} b_{i}^{m}+\delta b_{i}^{\text {noise }}
$$

In Eq. (10) $b_{i}^{m}$ are defined to be the button signals determined through the interpolation grid for the coordinates $(x, y)^{m}$ from Eq. (9). $g_{i}$ is the gain error on button $i$, and $t_{i}$ is an effective gain error for button $i$ arising from the timing error:

$$
t_{i}=1-\frac{a_{0}}{a_{2}+\frac{a_{1}^{2}}{4 a_{0}}}(\delta t[s])^{2}
$$

where the constants $a_{0,1,2}$ are empirically determined. Note that because CESR BPMs are timed to the peak signal of a bunch passage, any timing error will decrease the button signal. This method of modeling the timing error also allows the BPM model to account for synchrotron motion, thus modulating the timings on all four buttons on a turn-by-turn basis.

BPM position measurement reproducibility is dominated by electronic noise arising from the digitization and amplification of an analog signal on each of the four controllers, and is modeled in Eq. (10) as an additive error $\delta b_{i}^{\text {noise }}$ on each of the four button signals. The amplitude of the button-by-button reproducibility is set by determining the change in a single button signal consistent with changing the observed orbit by the desired reproducibility (for example $7 \mu \mathrm{m}$ ).

The top and bottom CESR BPM button blocks are welded separately to the vacuum chamber. There is then the possibility of a relative misalignment of the two blocks. In order to estimate the effect of this misalignment in simulation, upper and lower button signals are determined by offsetting the BPM in opposite directions.

\section{Simulation results}

Amplitudes for misalignments and BPM errors in the simulation are summarized in Tables VII, VIII, and IX, and are determined either from directly measured values or inferred from machine measurements. Offsets of quadrupoles and sextupoles include measured alignment levels 

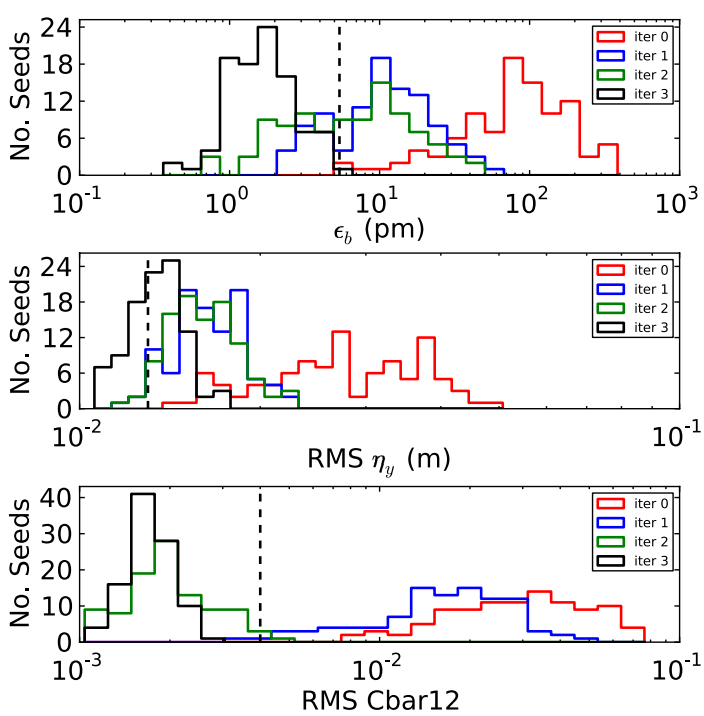

FIG. 4. Results from RING_MA2, using misalignments, BPM measurement errors, and multipoles stated in Tables VII, VIII, and IX, plotted before correction (red line), and after first, second, and third stage of emittance correction (blue, green, and black lines, respectively). The dashed black line indicates typical measured values in CESR after low-emittance correction.

along with $100 \mu \mathrm{m}$ added in quadrature to account for the estimated uncertainty in the offset of magnetic center with respect to geometric center of these elements.

The emittance correction procedure used in the simulation is identical to that used on the actual machine, outlined in Sec. VI. Results from RING_MA2 are shown in Fig. 4, and summarized in Table V.

After correction, 95\% of seeds achieved a vertical emittance below $4.1 \mathrm{pm}$, which is significantly smaller than the minimum measured vertical emittance of $10.3(+3.2 /-3.4)^{\text {sys }}( \pm 0.2)^{\text {stat }} \mathrm{pm}$ at $2.085 \mathrm{GeV}$. It is clear that the simulation does not account for more than half of the measured vertical emittance.

BPM tilts are the single most significant contribution to the vertical emittance in the simulation, and dominate the simulated $\eta_{y}$ measurement. Considering the simulation underestimates the measured vertical emittance, one could envision adjusting the simulation to reduce the amplitude of BPM tilts and increase magnet misalignments in order to increase the vertical emittance to levels measured in the actual machine while holding the rms $\eta_{y}$ constant. However, the required change in alignment to generate the measured vertical emittance is much larger than the measured uncertainty in the alignment procedure.

\section{DIAGNOSIS OF EMITTANCE DILUTION}

The two primary mechanisms for the static optics to contribute to vertical emittance are vertical dispersion and horizontal-to-vertical coupling. The measured vertical dispersion in Table II and the minimum $\bar{C}_{12}$ measured at
CesrTA $\left(2 \times 10^{-3}\right)$ are within the distributions from the simulation, indicating that the model is realistic. Increasing the coupling in simulated lattices such that the $\bar{C}_{12} \mathrm{rms}$ is consistent with the measurement in Table II introduces less than $1 \mathrm{pm}$ of vertical emittance.

Additionally, significant efforts have been made to ensure that all sources of uncertainty in the emittance measurement are accounted for. The discrepancy is therefore not attributed to emittance measurement errors. This implies there are significant sources of vertical emittance that are not included in the model or RING_MA2 simulation. Potential sources of vertical emittance are now considered.

\section{A. Emittance dilution from rf}

Random rf voltage and phase jitter may contribute to emittance dilution. There are four superconducting $\mathrm{rf}$ cavities in CESR, split into two pairs. Each pair is powered by a single power supply. Turn-by-turn beam size was recorded while varying the total rf voltage and number of $\mathrm{rf}$ cavities powered. The results are summarized in Table VI. It should be noted that the studies summarized in this section were taken while one of the two West rf cavities was disabled; therefore, only three rf cavities were used (one in the West, and two in the East). Nominal total rf voltage was 4.8 MV, distributed approximately evenly among the three cavities.

A small reduction in beam size was observed when reducing the total $\mathrm{rf}$ voltage from $4.8 \mathrm{MV}$ to $1.7 \mathrm{MV}$, corresponding to a reduction in observed vertical emittance of $0.3 \mathrm{pm}$. The $1 \sigma$ statistical uncertainty for the lowestmeasured emittance is $\pm 0.2 \mathrm{pm}$. Note that although the systematic uncertainty is an order of magnitude larger, it represents a global uncertainty where all measurements would be affected uniformly by any change in the understanding of the beam size measurement system.

A further reduction is seen when the single West rf cavity is powered down and detuned, such that only the two East rf cavities are running; the emittance increased slightly when running only on the $\mathrm{W} 1 \mathrm{rf}$ cavity. This indicates that the rf system is contributing to the vertical emittance, although the mechanism is not known at this time. The East and West rf cavity pairs run on separate power supplies; one hypothesis is that the West rf power supply is less stable than the East, thereby introducing vertical emittance through modulation of the rf voltage. Alternatively, by running a single cavity at a higher voltage, the amplitude of voltage jitter is also increased, potentially increasing the contribution to the emittance. The rf system in CESR is superconducting; therefore, a direct examination of the alignment requires the nontrivial process of warming the cavities and opening the cryostats.

\section{B. Collective effects}

The CesrTA emittance target of $10 \mathrm{pm}$ is for a "zerocurrent" beam, that is, neglecting any collective effects. 
TABLE V. 95\% C.L. correction levels after each correction iteration. All values except $\eta_{y}{ }^{\mathrm{Bmad}}$ include observational effects from BPM measurement errors. Details of the correction iterations are discussed in Sec. VI.

\begin{tabular}{lrrccc}
\hline \hline Measurement & Initial & Iter 1 & Iter 2 & Iter 3 & Units \\
\hline$\phi$ & 7.7 & 1.6 & 0.1 & 0.1 & $\mathrm{deg}$ \\
$\eta_{y}{ }_{\text {Meas }}$ & 42.6 & 18.7 & 18.7 & 15.4 & $\mathrm{~mm}$ \\
$\eta_{y}{ }^{\text {Bmad }}$ & 40.1 & 13.9 & 12.2 & 5.0 & $\mathrm{~mm}$ \\
$\bar{C}_{12}$ & 6.3 & 3.2 & 0.34 & 0.24 & $\times 10^{-2}$ \\
$\epsilon_{b}$ & 255.8 & 33.0 & 27.5 & 4.1 & $\mathrm{pm}$ \\
\hline \hline
\end{tabular}

TABLE VI. Summary of beam stability tests at CesrTA. The measurements were conducted in April 2013, for a single bunch of positrons at $0.7-0.85 \mathrm{~mA}$.

\begin{tabular}{lccc}
\hline \hline Total rf (MV) & East rf & West rf & $\epsilon_{b}(\mathrm{pm})$ \\
\hline 4.8 & On & On & 11.5 \\
1.7 & On & On & 11.2 \\
1.7 & Off & On & 12.5 \\
1.7 & On & Off & 10.8 \\
\hline \hline
\end{tabular}

Effects considered here include electron cloud, fast-ion instability, intrabeam scattering, and wakefields.

Electron cloud and fast-ion instability typically require a train of 30 bunches with $0.5 \mathrm{~mA} /$ bunch or more in order for emittance dilution to occur, and the emittance blowup takes place around bunch $10-15$ in the train [29]. A single bunch is not capable of generating sufficient cloud or ion density to cause emittance dilution.

Extensive measurements and simulations of intrabeam scattering at CesrTA indicate that the vertical emittance is largely insensitive to IBS effects at currents $I<1 \mathrm{~mA} /$ bunch, where the measurements reported here were taken $[30,31]$. The mechanism through which IBS increases vertical emittance depends on either transverseto-longitudinal scattering in regions with dispersion or transverse-to-transverse scattering in regions with coupling, such that the vertical-mode action of the particle changes. Vertical dispersion and coupling are measured to be globally well corrected, and are well below levels required for IBS to contribute to vertical emittance dilution.

Wakefields would tend to increase the emittance linearly with current. By measuring the dependence of the vertical beam size on current, it may be possible to determine whether wakefields are contributing to the emittance at the nominal $0.8 \mathrm{~mA} /$ bunch used for the emittance measurements presented in Table III. However, at such low current, photons are sparse and the turn-by-turn fitting procedure is no longer reliable. Instead, the turn-by-turn images must be averaged first in order to improve signal-to-noise, then fit as a single image. This has the disadvantages of incorporating a small amount of turn-by-turn beam motion and increasing the statistical uncertainty in the vertical emittance measurement.

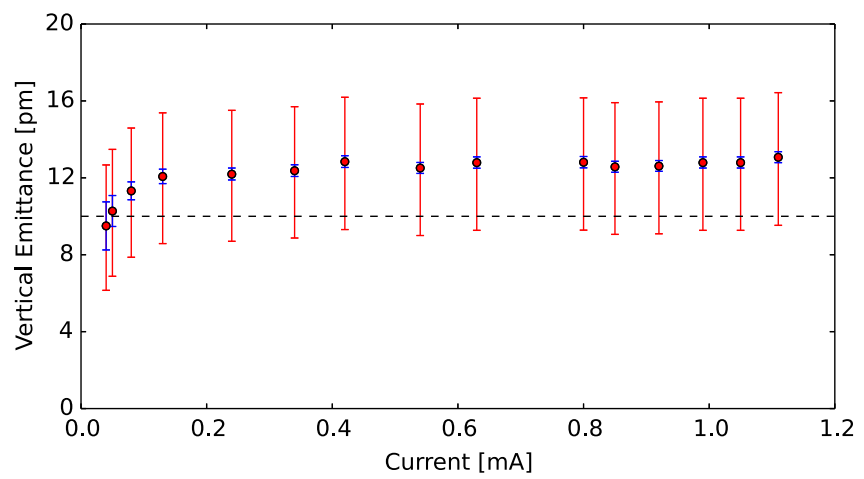

FIG. 5. Vertical emittance for a single bunch of positrons as a function of bunch current, from April 2013 CesrTA machine studies. Plotted error bars are systematic (red line) and statistical (blue line). The dashed horizontal line indicates the $10 \mathrm{pm}$ zero-current vertical emittance target for CesrTA.

Figure 5 shows the emittance calculated from a series of vertical beam size measurements from the XBSM, taken sequentially as the current was decreased from $1.1 \mathrm{~mA}$ to around $0.05 \mathrm{~mA}$, and processed as described above.

The rolloff of beam size at very low current $(<0.1 \mathrm{~mA})$ is likely due to lack of sufficient flux on the beam size monitor. Moreover, the emittance does not depend linearly on beam current. As such, there is no support for current-dependent effects diluting the vertical emittance at low-current.

\section{CONCLUSIONS}

A low-emittance tuning procedure has been developed at CesrTA, based on betatron phase and coupling measurements using resonant excitation and turn-by-turn capable BPMs. The tuning procedure has a fast turnaround, where one round of optics correction takes about $10 \mathrm{~min}$, and has yielded a single-bunch vertical emittance of $\epsilon_{b}=10.3$ $(+3.2 /-3.4)^{\text {sys }}( \pm 0.2)^{\text {stat }}$ pm with a single bunch of positrons with $0.8 \mathrm{~mA}=1.3 \times 10^{10}$ at $2.085 \mathrm{GeV}$. The correction procedure routinely achieves $\epsilon_{b}<15 \mathrm{pm}$ in a variety of machine conditions at energies ranging from $2.085-2.5 \mathrm{GeV}$.

The tuning procedure developed at CesrTA is significantly faster than response matrix analysis. The method scales independently of number of BPMs or correctors; thus for large machines the CesrTA procedure will be proportionally faster than response matrix analysis.

The lack of energy dependence for the minimumachieved vertical emittance may yield information regarding sources of emittance dilution. Further studies, including measuring the emittance at several energies during a single machine studies period, will be necessary before a conclusive statement may be made.

Collective effects do not appear to contribute to emittance dilution for a single bunch at $0.8 \mathrm{~mA}$. The rf system 
on the other hand clearly does affect the emittance, and further investigations are necessary.

Although misalignments do not appear to be the most significant contribution to the emittance, any improvement in alignment or optics correction will likely result in a small reduction in the emittance, as contributions to the emittance add linearly. In particular, BPM tilts remain a significant outstanding issue which limits the understanding of $\eta_{y}$. Simulations suggest that a reduction in rms BPM tilt from 12 to $5 \mathrm{mrad}$ could reduce the contribution of the static optics to the vertical emittance by $50 \%$. Alternative BPM tilt fitting techniques are under development.

\section{ACKNOWLEDGMENTS}

The authors wish to thank the CESR operations and instrumentation groups, whose support was indispensable in our efforts to achieve well-corrected conditions. This work was supported by the National Science Foundation Grant No. PHY-1002467 and the Department of Energy Grant No. DE-SC0006505.

\section{APPENDIX: ERRORS FOR RING_MA2 SIMULATIONS}

Table VIII shows the misalignments and errors used in CesrTA RING_MA2 studies. Offsets of quadrupoles and sextupoles include measured alignment levels along with $100 \mu \mathrm{m}$ added in quadrature to account for the estimated uncertainty in the offset of magnetic center with respect to geometric center of these elements.

Systematic multipoles are included for sextupoles that have vertical steering or skew quadrupole trim windings. These multipoles are computed using field modeling software and are scaled to a measurement radius of $20 \mathrm{~mm}$. There is a known random skew quadrupole component to the damping wiggler fields [32], due to manufacturing tolerances in the radii of the pole windings, which is also included. Multipoles used in this study are summarized in Table IX and use the following convention (summarized in the BMAD manual [33]):

$$
\frac{q L}{P_{0}}\left(B_{y}+\imath B_{x}\right)=\sum_{n=0}^{\infty}\left(b_{n}+\imath a_{n}\right)(x+\imath y)^{n},
$$

TABLE VII. BPM errors introduced into model CesrTA lattice for RING_MA2 studies.

\begin{tabular}{lcc}
\hline \hline Error & Applied rms & Units \\
\hline Reproducibility & 10 & $\mu \mathrm{m}$ \\
Tilt & 12 & $\mathrm{mrad}$ \\
Gains & $0.5 \%$ & $\%$ \\
Timing & 10 & $\mathrm{ps}$ \\
Offset $(x, y)$ & 170 & $\mu \mathrm{m}$ \\
Horizontal shear & \pm 100 & $\mu \mathrm{m}$ \\
\hline \hline
\end{tabular}

TABLE VIII. Misalignments and errors introduced into model CesrTA lattice for RING_MA2 studies. All parameters are determined either from machine measurements or survey.

\begin{tabular}{|c|c|c|c|}
\hline Element class & Error & rms & Units \\
\hline \multirow[t]{6}{*}{ Dipole } & $x$ offset & 0.9 & $\mathrm{~mm}$ \\
\hline & $y$ offset & 2.0 & $\mathrm{~mm}$ \\
\hline & $s$ offset & 2.3 & $\mathrm{~mm}$ \\
\hline & Roll & 144 & $\mu \mathrm{rad}$ \\
\hline & $x$ pitch & 600 & $\mu \mathrm{rad}$ \\
\hline & $y$ pitch & 300 & $\mu \mathrm{rad}$ \\
\hline \multirow[t]{8}{*}{ Quadrupole } & $x$ offset & 335 & $\mu \mathrm{m}$ \\
\hline & $y$ offset & 40.3 & $\mu \mathrm{m}$ \\
\hline & Magnetic offset & 100 & $\mu \mathrm{m}$ \\
\hline & $s$ offset & 5.2 & $\mathrm{~mm}$ \\
\hline & Tilt & 148 & $\mu \mathrm{rad}$ \\
\hline & $x$ pitch & 1100 & $\mu \mathrm{rad}$ \\
\hline & $y$ pitch & 62 & $\mu \mathrm{rad}$ \\
\hline & k1 & $0.1 \%$ & $\%$ \\
\hline \multirow[t]{8}{*}{ Sextupole } & $x$ offset & 280 & $\mu \mathrm{m}$ \\
\hline & $y$ offset & 280 & $\mu \mathrm{m}$ \\
\hline & Magnetic offset & 100 & $\mu \mathrm{m}$ \\
\hline & $s$ offset & 5.2 & $\mathrm{~mm}$ \\
\hline & Tilt & 200 & $\mu \mathrm{rad}$ \\
\hline & $x$ pitch & 1200 & $\mu \mathrm{rad}$ \\
\hline & $y$ pitch & 800 & $\mu \mathrm{rad}$ \\
\hline & $k 2$ & $0.1 \%$ & $\%$ \\
\hline \multirow[t]{6}{*}{ Wiggler } & $x$ offset & 1 & $\mathrm{~mm}$ \\
\hline & $y$ offset & 250 & $\mu \mathrm{m}$ \\
\hline & $s$ offset & 500 & $\mu \mathrm{m}$ \\
\hline & Tilt & 300 & $\mu \mathrm{rad}$ \\
\hline & $x$ pitch & 200 & $\mu \mathrm{rad}$ \\
\hline & $y$ pitch & 250 & $\mu \mathrm{rad}$ \\
\hline
\end{tabular}

where $b_{n}$ and $a_{n}$ are the normal and skew multipoles, respectively. The values in the table are normalized by $1 /\left(K_{m} L r_{0}^{m}\right)$, where $m$ is the order of the primary field ( $m=1$ for quadrupole, 2 for sextupole, etc.).

TABLE IX. Multipoles used in RING_MA2 studies of CesrTA lattice. Sextupole multipoles are systematic and therefore identical at all sextupoles, whereas the wiggler $a 1$ multipole is random; the number quoted for wiggler $a 1$ is therefore the rms of the applied distribution.

\begin{tabular}{lcc}
\hline \hline Element class & Multipole & Value \\
\hline Sextupole with & $a 3$ & $-7.25 \times 10^{-4}$ \\
vertical steering trim & $a 5$ & $-1.46 \times 10^{-2}$ \\
& $a 7$ & $6.68 \times 10^{-4}$ \\
& $a 9$ & $8.7 \times 10^{-6}$ \\
Sextupole with & $a 11$ & $1.0 \times 10^{-5}$ \\
skew quad trim & $a 4$ & $-1.2145 \times 10^{-1}$ \\
& $a 6$ & $2.16 \times 10^{-4}$ \\
& $a 8$ & $4.96 \times 10^{-4}$ \\
Wiggler & $a 10$ & $-2.29 \times 10^{-5}$ \\
\hline \hline
\end{tabular}


[1] M. A. Palmer et al., in Proceedings of the 23rd Particle Accelerator Conference, Vancouver, BC, 2009 (IEEE, Piscataway, NJ, 2009), pp. 4200-4204.

[2] G. F. Dugan, M. A. Palmer, and D. L. Rubin, in ICFA Beam Dynamics Newsletter, edited by J. Urakawa (International Committee on Future Accelerators, Batavia, IL, 2009), pp. 11-33.

[3] D. Rubin, D. Sagan, and J. Shanks, in Proceedings of the 23rd Particle Accelerator Conference, Vancouver, BC, 2009 (IEEE, Piscataway, NJ, 2009), pp. 2751-2753.

[4] “The International Linear Collider Technical Design Report," ILC Global Design Effort, 2013.

[5] J. Safranek, Nucl. Instrum. Methods Phys. Res., Sect. A 388, 27 (1997).

[6] J. Safranek, G. Portmann, A. Terebilo, and C. Steier, SSRL/SLAC and LBNL "MATLAB-Based LOCO," Technical Report, 2002.

[7] M. Aiba, M. Boge, N. Milas, and A. Streun, Nucl. Instrum. Methods Phys. Res., Sect. A 694, 133 (2012).

[8] R. Dowd, M. Boland, G. LeBlanc, and Y.-R. E. Tan, Phys. Rev. ST Accel. Beams 14, 012804 (2011).

[9] A. Wolski, Phys. Rev. ST Accel. Beams 9, 024001 (2006).

[10] H. Qin, R. C. Davidson, M. Chung, and J. W. Burby, Phys. Rev. Lett. 111, 104801 (2013).

[11] C. Baumgarten, Phys. Rev. ST Accel. Beams 15, 124001 (2012).

[12] D. Edwards and L. Teng, IEEE Trans. Nucl. Sci. 20, 885 (1973).

[13] D. Sagan and D. Rubin, Phys. Rev. ST Accel. Beams 2, 074001 (1999).

[14] M. A. Palmer et al., in Proceedings of the 2010 International Particle Accelerator Conference, Kyoto, Japan (ICR, Kyoto, 2010), pp. 1191-1193.

[15] D. Sagan, R. Meller, R. Littauer, and D. Rubin, Phys. Rev. ST Accel. Beams 3, 092801 (2000).

[16] R. E. Meller and M. A. Palmer, in Proceedings of the 24th Particle Accelerator Conference, PAC-2011, New York (IEEE, New York, 2011), pp. 504-506.

[17] D. L. Rubin, M. Billing, R. Meller, M. Palmer, M. Rendina, N. Rider, D. Sagan, J. Shanks, and C. Strohman, Phys. Rev. ST Accel. Beams 13, 092802 (2010).

[18] Y. Chung, D. Barr, G. Decker, K. Evans, Jr, and E. Kahana, in Proceedings of the Particle Accelerator Conference, Dallas, Texas, 1995 (IEEE, New York, 1995), Vol. 4, pp. 2452-2454.

[19] F. Tecker, B. Dehning, P. Galbraith, K. Henrichsen, M. Placidi, and R. Schmidt, in Particle Accelerator
Conference, Vancouver, BC, 1997 (IEEE, New York, 1997), Vol. 3, pp. 3648-3650.

[20] J. P. Shanks, D. Rubin, and D. Sagan, in Proceedings of the 2010 International Particle Accelerator Conference, Kyoto, Japan (ICR, Kyoto, 2010), pp. 4620-4622.

[21] N. T. Rider, J. P. Alexander, J. A. Dobbins, M. G. Billing, R. E. Meller, M. A. Palmer, D. P. Peterson, C. R. Strohman, and J. W. Flanagan, in Proceedings of the 24th Particle Accelerator Conference, PAC-2011, New York (IEEE, New York, 2011), pp. 687-689.

[22] N. T. Rider, M. G. Billing, M. P. Ehrlichman, D. P. Peterson, D. Rubin, J. P. Shanks, K. G. Sonnad, M. A. Palmer, and J.W. Flanagan, in Proceedings of IBIC 2012: International Beam Instrumentation Conference, Tsukuba, Japan (JACoW, Geneva, Switzerland, 2012), Paper WECD01, pp. 585-589.

[23] J. P. Alexander, A. Chatterjee, C. Conolly, E. Edwards, M. P. Ehrlichman, E. Fontes, B. K. Heltsley, W. Hopkins, A. Lyndaker, D. P. Peterson, N. T. Rider, D. L. Rubin, J. Savino, R. Seeley, J. Shanks, and J. W. Flanagan, Nucl. Instrum. Methods Phys. Res., Sect. A 748, 96 (2014).

[24] J. Shanks et al., in Proceedings of the 24th Particle Accelerator Conference, PAC-2011, New York (IEEE, New York, 2011), pp. 1540-1542.

[25] J.P. Shanks et al., in Proceedings of the 23rd Particle Accelerator Conference, Vancouver, BC, 2009 (IEEE, Piscataway, NJ, 2009), pp. 2754-2756.

[26] A. Wolski, D. Rubin, D. Sagan, and J. Shanks, Phys. Rev. ST Accel. Beams 14, 072804 (2011).

[27] D. Sagan, Nucl. Instrum. Methods Phys. Res., Sect. A 558, 356 (2006).

[28] R. W. Helms and G. H. Hoffstaetter, Phys. Rev. ST Accel. Beams 8, 062802 (2005).

[29] M. Billing et al., in Proceedings of the 4th International Particle Accelerator Conference, IPAC-2013, Shanghai, China (JACoW, Shanghai, China, 2013), pp. 1844-1846.

[30] M. Ehrlichman, Ph.D. thesis, Cornell University, Ithaca, New York, 2013.

[31] M.P. Ehrlichman et al., in Proceedings of the 3rd International Particle Accelerator Conference, New Orleans, LA, 2012 (IEEE, Piscataway, NJ, 2012), pp. 2970-2972.

[32] J. A. Crittenden, A. Mikhailichenko, E. Smith, K. Smolenski, and A. Temnykh, in Proceedings of the 21st Particle Accelerator Conference, Knoxville, TN, 2005 (IEEE, Piscataway, NJ, 2005), pp. 2336-2338.

[33] D. Sagan, The Bmad Reference Manual (CLASSE, Ithaca, NY, 2013). 